\title{
Avoiding Extraverts: Pathogen Concern Downregulates Preferences for Extraverted Faces
}

\author{
Mitch Brown $^{1}$ • Donald F. Sacco ${ }^{1}$
}

Published online: 2 August 2016

(C) Springer International Publishing 2016

\begin{abstract}
Past research indicates that salient concerns with infectious disease reduce individuals' self-reporting of extraverted personality trait characteristics, an adaptive response to mitigate exposure to pathogenically threatening conspecifics. Additionally, individuals are capable of accurately inferring another person's level of extraversion from facial cues alone. Extending these findings, we hypothesized that disease concerns should result in a reduced preference for extraverts, as indexed by facial cues, given that such persons may comprise a greater disease threat due to increased contact with a greater number of conspecifics. To test this hypothesis, participants were randomly assigned to either disease or control prime conditions, reported face preferences among face pairs containing target faces manipulated to communicate extraversion or introversion, and indicated dispositional pathogen concerns. Contrary to hypotheses, acute disease activation did not influence face preferences. However, men with dispositionally higher perceived infectability (PI) demonstrated reduced preferences for extraverted female faces, whereas higher PI in women predicted a reduced preference for extraverted male faces. This relationship between higher PI and reduced preferences for extraverted faces provides partial support for the hypothesis that pathogen concerns facilitate stronger preferences for reticent individuals, an adaptive response to mitigate contact with disease vectors.
\end{abstract}

Keywords Extraversion $\cdot$ Disease $\cdot$ Personality $\cdot$ Face perception $\cdot$ Perceived infectability

Mitch Brown

mitchellbrown@eagles.usm.edu

1 Department of Psychology, The University of Southern Mississippi, Owings-McQuagge Hall 226, Hattiesburg, MS 39406, USA
When choosing friends, group members, or relationship partners, selection typically favors those appearing friendly and gregarious. Such extraverted persons likely appear more interested in affiliation. It may thus seem advantageous to include extraverts in group activities over those more interpersonally restricted (i.e., introverts) because of their greater potential in satisfying affiliative needs. Despite potential adaptive advantages for extraverts in group living, the heightened interpersonal contact associated with extraversion, due to their extensive social networks (Pollett, Roberts, and Dunbar 2011), may regularly expose extraverts to more environmental pathogens than would the socially restricted behavior of reticent individuals. This would thus implicate extraverted individuals as greater risks for infectious disease exposure and transmission. Indeed, past research indicates that salient disease concerns lead individuals to report themselves as having a less extraverted personality as well as demonstrate greater behavioral reticence (Mortensen et al. 2010), which would be adaptive responses to effectively reduce contact with others who may be vectors for infectious pathogens. In the current study, we sought to expand on these findings and hypothesized that concerns with disease exposure would elicit reduced preferences for others who communicate an extraverted disposition, as communicated via structural facial cues.

\section{Pathogen Threat and Personality}

Environmental pathogens exert a pervasive influence over several Big Five Personality traits implicated in facilitating sociality, including extraversion. This is sensible, given that the benefits and costs of extraversion vary based on pathogenic threat in one's environment. For example, cross-cultural 
research finds higher levels of historical pathogen prevalence in a given culture predict reduced extraversion at the population level (as well as reduced openness to experience and more restricted sociosexuality; Schaller and Murray 2008; Thornhill et al. 2010). These environmental pathogen-contingent fluctuations in extraversion (i.e., greater levels of extraversion in low pathogen environments versus reduced levels of extraversion in high pathogen environments) would thus be adaptive to navigate the potential benefits and costs of extraversion. High levels of extraversion would facilitate interpersonal behavior that would satisfy social affiliation, which is of inherent importance to human survival. Indeed, gregariousness is instrumental in forming social bonds and creating a cooperative social network (Pollett et al. 2011). Thus, in low-pathogen environments, one can more safely access the benefits of extraversion, without the potential costs, because overall pathogen contamination likelihood is low. However, the more frequent contact with a greater number of conspecifics seen among extraverted individuals necessarily increases one's exposure to pathogenic threats. For example, the expanded social networks of extraverted person is directly related to greater likelihood of contracting illnesses (e.g., upper respiratory infections; Hamrick, Cohen, and Rodriguez 2002; Nettle 2005). Thus, in high-pathogen environments, other individuals represent a significant communicable disease threat; being highly extraverted in this kind of environment would be inherently risky, making low levels of extraversion more adaptive (even though being low in extraversion could mean missing out on social opportunities). The findings that the expression of extraverted personality is contingent on local pathogen threat are thus quite adaptive in the context of managing the benefits and costs of being extraverted.

Corroborating these findings, experimental research suggests that acutely activated disease threat produces similar reticence in personality. Mortensen et al. (2010) primed participants with disease concerns (or a negative control experience) and subsequently assessed their self-identification with Big Five traits on a situational level. Individuals primed with disease reported lower extraversion. Furthermore, those with dispositionally higher perceived vulnerability to disease reported lower levels of agreeableness and openness to experience following such priming; neuroticism and conscientiousness were unaffected. These results suggest reticence would provide a considerable adaptive advantage for individuals in highly pathogenic environments. Such restricted social behavior would limit the physical proximity required for communicable disease transmission. Additionally, these findings suggest that individual differences in self-perceptions of vulnerability to disease and acutely activated disease concerns can interact to influence adaptive behavior.

Although findings suggest that salient disease concerns influence one's own levels of extraversion, research has yet to determine how such concerns influence evaluations of others based on their levels of extraversion. Given that individuals themselves become less extraverted when disease concerns are heightened to reduce their own exposure to pathogenic conspecifics, they should also demonstrate a preference for others who communicate a similarly reticent personality, as these individuals would likely be less of a disease threat themselves due to their own restricted social network. Nonetheless, for this adaptive preference to be possible, individuals would need to be sensitive to cues in others indicative of relative health or disease threat, particularly when they perceive themselves to be at risk for pathogen exposure.

\section{The Behavioral Immune System and Detecting Pathogenic Threat in Conspecifics}

To conserve resources in the biological immune system, it has been argued that humans evolved a behavioral immune system (BIS) that activates upon exposure to cues of pathogenic threat. This activation results in perceptual, cognitive, emotional, and behavioral reactions designed to preemptively detect, and subsequently avoid, potentially pathogenic conspecifics (Neuberg, Kenrick, and Schaller 2011; Schaller and Duncan 2007). Importantly, humans may evaluate conspecifics through various physical features implicated in communicating others' potential pathogen load upon BIS activation. These features are ostensibly those associated with targets' relative overall physical health. Categorization of targets as disease vectors is typically based on facial disfigurement (e.g., Ackerman et al. 2009), old age (Duncan and Schaller 2009), and obesity (e.g., Miller and Maner 2012). BIS activation elicits both greater perceptual acuity in detecting pathogen cues in social targets (Miller and Maner 2012), and the subsequent behavioral aversion of targets who possess such cues (Mortensen et al. 2010).

Along with cues suggesting greater disease threat, other physical features communicate reduced threat or less prior exposure to disease vectors, subsequently resulting in heightened preference for these features. For example, greater facial symmetry is associated with lower mutation load and greater disease resistance (e.g., Gangestad, Thornhill, and Yeo 1994). Research further indicates that BIS activation (both dispositional and situational) produces augmented preferences for facial symmetry (Little, DeBruine, and Jones 2011; Young, Sacco, and Hugenberg 2011). This identification and subsequent preference for these faces would be adaptive in identifying, and approaching, ostensibly physically healthier conspecifics.

Though physical facial structures may connote underlying disease resistance and mutation load (e.g., facial symmetry), other facial features may provide similar information regarding targets' behavioral tendencies that would influence overall exposure to pathogenic threat. That is, certain facial features may connote an individual's level of extraversion, which 
would be a cue to the overall social contact they have had with other conspecifics. Previous research has elucidated that facial morphology can signal aspects of an individual's personality (e.g., Holtzman 2011; Little and Perrett 2007), which would be informative about their behavioral tendencies. That inference would be beneficial, as an individual would want to identify and associate with conspecifics best able to aid in satisfying salient needs. If such needs concern environmental pathogens, individuals with personalities tied to reduced pathogen load (i.e., introversion) would likely be preferred. We hypothesize that to the extent that individuals can accurately detect a social target's extraversion from facial cues, disease concerns should lead to a reduced preference for extraverted social targets (much as such concerns reduce an individual's own personal endorsement of extraverted personality traits).

\section{Extraversion Detection from Facial Cues}

Research suggests a reliable degree of accuracy for humans' identification of personality traits based on facial features (e.g., Petrican, Todorov, and Grady 2014). For example, people are rather adept at detecting targets' trait levels of conscientiousness and extraversion based on images of target persons. When rating the degree to which social targets exhibited Big Five traits based on brief exposure to faces of individuals who had previously completed personality inventories (50$150 \mathrm{~ms}$ ), participants' assessments correlated strongly with targets' self-reported exhibition of traits (Borkenau et al. 2009). Importantly, this relation was strongest for perceptions of extraversion. These findings suggest that individuals are capable of efficiently and accurately extracting information about a social target's relative level of extraversion from characteristics of their face. As such, when confronted with individuals' faces, salient disease concerns should lead individuals to demonstrate reduced preferences for extraverted social targets, which would complement past research demonstrating disease salience reduced self-reported extraversion (Mortensen et al. 2010).

\section{The Current Study}

The goal of the current study was to identify how BIS activation influences person perception as it relates to the communicative properties of extraversion and introversion in human faces. Whereas previous research has demonstrated that individuals are relatively accurate at detecting extraversion from faces (e.g., Borkenau et al. 2009), it has yet to indicate when this identification elicits preference or aversion of targets based on these detected levels of extraversion. Given that past research demonstrates, specifically, that acute activation of disease concerns leads to increased self-reported reticence, particularly among those with dispositionally higher concern with disease (Mortensen et al. 2010), we tested the following hypotheses with respect to preferences for reticence in others based on facially communicated extraversion and introversion: (1) acute BIS activation via disease priming would result in reduced preferences for extraverted faces, and (2) this would be especially pronounced among individuals with higher dispositional concerns with disease (i.e., perceived vulnerability to disease).

\section{Method}

\section{Participants}

One hundred fifty-five ( 81 men, 74 women; $M_{\text {Age }}=35.63$ years, $\mathrm{SD}=12.05 ; 88.3 \%$ White) individuals participated through Amazon's Mechanical Turk (Buhrmester, Kwang, and Gosling 2011) survey tool in exchange for US $\$ 0.35$. A medium-effect size power analysis (Cohen's $f=0.25, \beta=0.80$; Faul et al. 2007) indicated that 128 participants were needed to detect effects; we intentionally oversampled.

\section{Materials}

Priming Task Participants were randomly assigned to one of two priming conditions in which they viewed a slideshow to make either pathogen concern (disease prime) or general negative affect (control) salient. Participants viewing the disease prime saw 10 images for $6 \mathrm{~s}$ each of persons exhibiting various disease symptoms (e.g., chicken pox, gangrenous toes). Participants in the control condition saw 10 images of individuals "pointing" guns at them to prime negative affect unrelated to pathogen concern. Both slide shows were borrowed from previous research designed to activate disease concerns versus a negative control state (Schaller et al. 2010).

Following the priming slideshows, participants completed a 12-item manipulation check questionnaire derived from Schaller et al. (2010). This measure operated on 7-point Likert-type scales $(1=$ Not at All, $7=$ Very Much $)$ along 4 subscales (4 questions each) assessing the extent to which the participants experienced each feeling at that very moment: Tension (e.g., "How tense do you feel at this very moment?"), Peace (e.g., "How calm do you feel at this very moment?"), Disgust (e.g., "How disgusted do you feel at this very moment?"), and Fear (e.g., "How fearful do you feel at this very moment?"), with higher scores indicating higher degree of experiencing the designated affective response following the slide show. All subscales demonstrated acceptable reliabilities ( $\left.\alpha_{\mathrm{S}}>0.94\right)$. 


\section{Materials}

Introversion-Extraversion Faces To create faces communicating introversion and extraversion, we selected 20 male and 20 female Caucasian faces that originated from both the Aging Faces (Minear and Park 2004) and Chicago Face Databases (Ma, Correll, and Wittenbrink 2015) with target individuals' ages ranging from 18 to 40 years. Each target was morphed with a composite face prototype created by Holtzman (2011) to communicate both high- and low-extraversion (i.e., introversion) faces from a collection of personality-derived faces in a "Faceaurus." Specifically, individuals completed the Big Five Personality trait inventory, and then a composite extraversion face was generated by averaging the faces of the ten individuals who scored highest in extraversion in their sample; conversely, a composite "introversion" face was created by averaging the faces of the ten individuals scoring lowest in extraversion (based on a combination of self- and peer-reported extraversion/introversion scores; Holtzman 2011). There was a prototype of the average extraverted and introverted face for each sex.

Using these four composite faces (i.e., female extravert, female introvert, male extravert, male introvert), we blended each target face with the matched-sex extravert and introvert prototype using morphing software (Morpheus Animation Suite v3.10), such that morphs were $50 / 50$ blends (i.e., $50 \%$ original face $/ 50 \%$ prototype). For each male and female target face, we created an extraversion and introversion face. This resulted in 40 face pairs, or 20 female and 20 male targets with an extraversion and introversion version of the original faces (see Appendix for sample face pairs).

Participants were randomly presented with male and female morphed face pairs, with one of the images being high extraversion morph of the target and one of the images being the high introversion morph of the target (location of introversion and extraversion morph counterbalanced on a betweenparticipants basis). On each trial, participants were asked to select the version of the face that they preferred. The task was self-paced, such that each face pair was displayed until participants generated a preference response; upon responding, participants saw the next face pair. To calculate participants' preference for extraverted face morphs (relative to introverted face morphs), we summed the number of times participants selected the extraversion morph and divided it by the total number of trials, separately for male and female targets, to create an extravert preference ratio; higher values reflected a larger preference for extraverted faces.

Perceived Vulnerability to Disease Participants then completed the 15-item Perceived Vulnerability to Disease scale
(PVD; Duncan, Schaller, and Park 2009), which consists of two subscales for perceived infectability and germ aversion to tap unique aspects of dispositional BIS responses. The Perceived Infectability (PI) subscale contains 7 items and taps an individual's beliefs that they are personally susceptible to contagious disease (e.g., "If an illness is 'going around,' I will get it."). The Germ Aversion (GA) subscale contains 8-items and taps into individuals' emotional aversion to pathogen threats (e.g., "It does not make me anxious to be around sick people." [reverse-scored]). Participants responded using a 7 point Likert-type scale $(1=$ Strongly Disagree; $7=$ Strongly Agree) with higher scores indicating greater perceptions of vulnerability to disease; 6 items required reverse-scoring. Both PI $(\alpha=0.83)$ and GA subscales $(\alpha=0.75)$ reported acceptable reliabilities.

\section{Procedure}

Interested participants viewed a description of the study through MTurk and clicked a link redirecting them to the consent form, from which they clicked a link to the study. Participants were first randomly assigned to either view the disease prime or control slide show. All participants then completed the slide show manipulation check, followed by the introversion-extraversion face preference task, and the PVD scale. Finally, participants completed a demographics form (e.g., age, gender) and were redirected to a debriefing page. They received a six-digit code redeemable for study payment.

\section{Results}

\section{Manipulation Check}

To determine the effectiveness of our manipulation, we ran 4 independent samples $t$ tests, with condition as the independent variable for feelings of Tension, Peace, Disgust, and Fear. Disease-primed participants reported greater tension $(M=4.29, \mathrm{SD}=1.85)$ than did control prime $(M=3.05$, $\mathrm{SD}=1.79), t(153)=4.24, p<0.001, d=0.68$. Disease-primed participants reported less peacefulness $(M=3.23, \mathrm{SD}=1.77)$ than did control $(M=3.84, \mathrm{SD}=1.81), t(153)=-2.15$, $p=0.033, d=0.34$. Disease-primed participants reported greater disgust $(M=4.19, \mathrm{SD}=2.07)$ than did control $(M=3.15, \mathrm{SD}=2.18), t(153)=3.03, p=0.003, d=0.49$. Disease-primed reported greater fear $(M=3.06, \mathrm{SD}=1.98)$ than did control $(M=2.41, \mathrm{SD}=1.65), t(153)=2.24$, $p=0.027, d=0.35$. These results, particularly for the disgust subscale, indicate that the disease prime manipulation was effective in activating disease concern. 


\section{Preliminary Face Analyses}

Before conducting our primary analysis, we conducted one-sample $t$ tests to determine the extent to which individuals demonstrate a relative preference for introverted or extraverted faces. Since 0.5 would indicate chance responding for face preferences (i.e., choosing one face type or another $50 \%$ of the time), that was our comparison point for targets. Participants significantly preferred communicated extraversion in faces overall $(M=0.56$, $\mathrm{SD}=0.09), t(154)=8.95, p<0.001, d=1.44$. When target sex was considered, there was a significantly greater-thanchance preference for extraverted female targets $(M=0.62, \mathrm{SD}=0.12), t(154)=11.99, p<0.001, d=1.93$; however, there was no preference for either extraversion or introversion in male target faces $(M=0.51, \mathrm{SD}=0.13)$, $t(154)=0.95, p=0.342, d=0.15$. Although there was a significant overall preference for faces communicating extraversion, this finding was driven by female targets. We offer a tentative explanation for this preference independent of pathogen concern in the "Discussion."

\section{Primary Analysis}

To test the hypothesis that dispositional disease concerns would interact with disease threats to influence preferences for extraversion in faces, we conducted separate analyses for Perceived Infectability (PI) and Germ Aversion (GA) subscales in the context of acute disease concerns. Specifically, past research demonstrates that the GA and PI are associated with different kinds of perceptual and behavioral outcomes (e.g., Duncan and Schaller 2009; Young et al. 2011); therefore, we analyzed their impacts on face preferences separately. Importantly, though correlated, both subscales' association to each other was relatively small in the current study, $r(154)=0.24$, $p=0.003$, which suggests that they are tapping unique dimensions of PVD that would otherwise not be detected if they were collapsed onto each other. Additionally, given that past research shows PI (but not GA) is related to adaptive face preferences (i.e., symmetry preferences; Young et al. 2011), we hypothesized that PI would be more likely to be related to face preferences for extraversion/introversion than would GA. However, we still included analyses with the GA subscale to be comprehensive in our analyses and consistent with previous research.

Perceived Infectability We initially conducted a 2 (Condition: Disease vs. Control) $\times 2$ (Participant Sex: Male vs. Female $) \times 2$ (Target Sex: Male vs. Female) mixed-model ANCOVA, with repeated measures over Target Sex, and participants' PI scores as a covariate to test for interactive effects of PI with other model variables. Contrary to our hypothesis, there was no main effect of Condition and Condition did not interact with any other variables in the model (all $p s>0.192$ ); thus, acute activation of disease concerns did not influence preferences for introverted or extraverted faces. There was a marginally significant main effect of PI such that higher levels of PI were associated with reduced preferences for extraversion in faces, $F(1,147)=3.48, p=0.060$, $\eta_{p}{ }^{2}=0.024$. Importantly, there was a marginally significant three-way interaction between participant sex, target sex, and PI, $F(1,147)=3.81, p=0.053, \eta_{p}{ }^{2}=0.025^{1}$.

To best understand this interaction, we conducted two separate regression analyses for extraversion preferences using PROCESS Model 1 in SPSS (see Hayes 2013) to conduct simple slope analyses. PI and Participant Sex were the independent variables in each model: one model tested for female target extraversion preferences and the other model for male target extraversion preferences. In these analyses, Participant Sex was dummy-coded such that men were coded as a " 0 " (reference group) and women as a "1." This analysis revealed a marginally significant PI $\times$ Target Sex interaction, $\Delta R^{2}=0.023, b=0.032$, $\mathrm{SE}=0.02, t(154)=1.92, p=0.056$. To understand this interaction, we utilized simple slopes analyses to determine where a relation could have emerged. Women's PI scores were unrelated to their extraversion preferences in female faces, $b=0.006, \mathrm{SE}=0.01, t(151)=0.54, p=0.582$. However, for men, there was a significant negative relation between PI and extraversion preferences in female faces such that a one-unit increase in men's PI results in a 0.025 -unit reduction in extravert preference, $b=-0.025$, $\mathrm{SE}=0.01, t(151)=-2.08, p=0.039$. In other words, men with higher dispositional perceptions of being susceptible to infectious disease had a greater preference for women's faces that communicated introversion, relative to extraversion (see Fig. 1).

The second analysis was for preferences in male faces. This analysis yielded no significant main effects or interactions (all $p \mathrm{~s}>0.115$ ). However, to have a full understanding of our data, we thought it prudent to analyze the simple effects for either sex and determine if any relationship may have emerged. For male participants, there was no significant relation between PI and extraversion preference in male faces, $b=-0.002, \mathrm{SE}=0.01$, $t(80)=-0.18, p=0.857$. Among women, however, there was a marginally significant negative relation between

\footnotetext{
${ }^{1}$ Because condition (disease prime, control prime) produced no main effects or interactions when included in the omnibus analysis, we conducted a supplemental analysis in which this variable was excluded. Importantly, in this analysis, the critical three-way interaction between participant sex, perceived infectability, and target sex reached conventional significance, $F(1,151)=4.49, p=0.036, \eta_{p}^{2}=0.029$.
} 


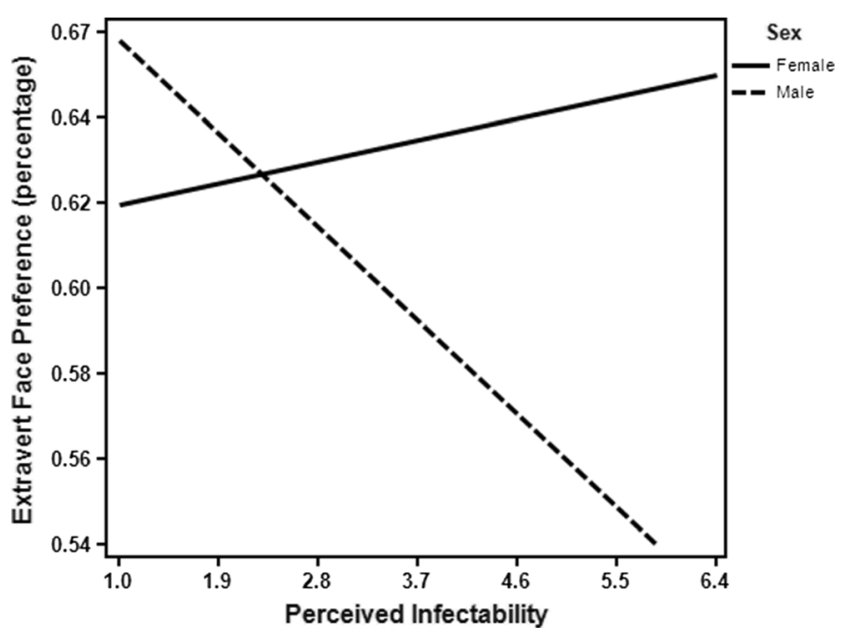

Fig. 1 Interaction plot for female extravert preference as a function of participant sex and perceived infectability

PI and male face preference such that a one-unit increase in PI results in a 0.020 -unit reduction in extraversion preference for male faces, $b=-0.020, \mathrm{SE}=0.01, t(73)=-1.83$, $p=0.068$. In other words, PI marginally predicts a preference for introverted male faces among women. This effect did not attain conventional significance, but this relation was consonant with the previous analysis in terms of PI predicting cross-sex preference for introversion (see Fig. 2).

Germ Aversion We conducted a second 2 (Condition: Disease vs. Control) $\times 2$ (Participant Sex: Male vs. Female $) \times 2$ (Target Sex: Male vs. Female) mixed model ANCOVA, with repeated measures over Target Sex and participants' GA scores as a covariate to test for interactive effects between GA and the disease prime. Contrary to hypotheses,

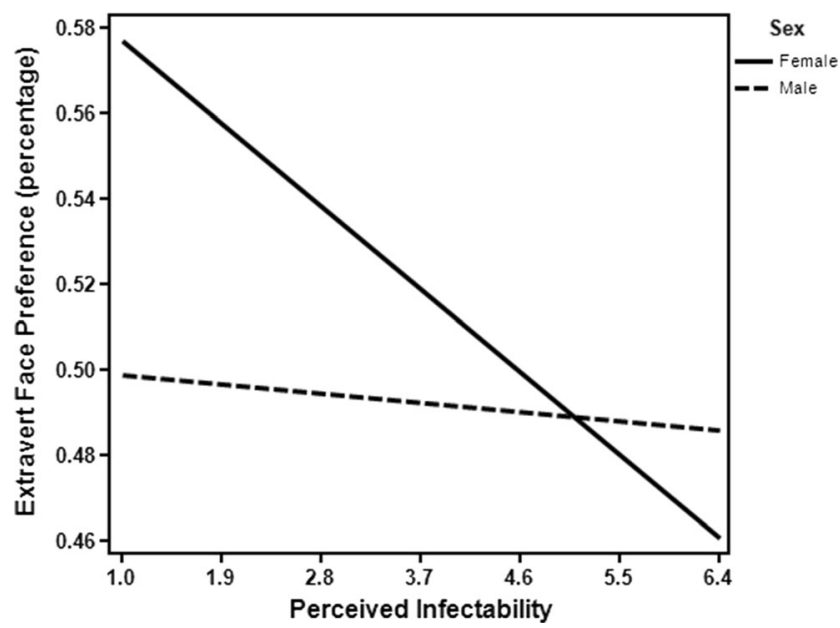

Fig. 2 Interaction plot for male extravert preference as a function of participant sex and perceived infectability there was no main effect of condition, nor did condition interact with any other variables in the model (all $p \mathrm{~s}>.280$ ); thus, acute activation of disease concerns did not influence preferences for introverted or extraverted faces. Furthermore, no effects that emerged were related to GA $(p s>0.118)$. Thus, we did not probe this analysis any further.

\section{Discussion}

These findings provide preliminary evidence for how BIS activation influences face perception in the context of facial cues associated with extraversion, albeit only in terms of dispositional activation of pathogen concern, as acute activation of pathogen threat did not predict face preferences in this study. Humans exhibit proficiency at initially perceiving personality through faces such that they can accurately determine whether someone is an introvert or extravert from facial characteristics alone (Borkenau et al. 2009). Our results extend these findings to demonstrate motivation-influenced preference shifts for extraverted personality traits, as communicated by facial cues. There was also a marginal relation between higher perceived infectability and a reduced preference for extraverted targets for both men and women, suggesting that those with chronic infection concerns show a reduce preference for extraverted others. Given that extraverted others have larger social networks and are more frequently exposed to pathogens (Hamrick et al. 2002), it would be adaptive for those with poorer self-perceived immunocompetence to avoid targets whose traits suggest greater communicable disease threat (i.e., extraverted people). Even though this would result in potentially missing greater social opportunities by not associating with extraverted others, the pathogen costs associated with extraverted targets would de-emphasize the importance of affiliation to high-PI individuals.

Nonetheless, the relation between perceived infectability and extraversion preferences was qualified by both participant and target sex. Specifically, men higher in dispositional perceived infectability demonstrated a reduced preference for extraverted female targets, whereas women higher in perceived infectability demonstrated a reduced preference for extraverted male faces. These preferences for introverted targets among those higher in perceived infectability are adaptive. Compared to extraverted persons, introverted individuals would have been less likely to encounter disease due to exposure to fewer conspecifics, therefore posing less disease threat. 
We originally predicted that greater concern with pathogen threat would reduce preferences for extraverted targets, regardless of target sex. However, the fact that this preference shift primarily occurred for cross-sex faces (i.e., men's preferences for female faces and women's preferences for male faces) suggests that it may be especially related to preventing contact with potentially pathogenic mating partners. Greater environmental pathogen loads foster more restrictive sexual strategies (Murray, Jones, and Schaller 2013; Schaller and Murray 2008), which should thus make individuals who pose reduced infection risk especially desirable among those with greater pathogen concern (e.g., Gangestad and Buss 1993). Since intimate contact is highly associated with communicable disease transmission (e.g., STIs; Halperin and Epstein 2004), a preference for sexually reticent others in the context of mate selection may be especially important, as opposed to general affiliation. Facially communicated introversion may indicate a prospective mate as posing less disease risk. Furthermore, and germane to mating domains, extraversion is associated with unrestricted sexual strategies (Nettle 2005; Schmitt and Shackelford 2008). That is, extraverted individuals exhibit greater interest in using promiscuous sexual strategies, compared to introverted individuals, which may ultimately lead to their overall chance of illness (Hamrick et al. 2002; Nettle 2005). Someone with heightened perceived infectability may recognize extraverted individuals of the opposite sex as potentially more behaviorally promiscuous and therefore down-regulate their interest in them.

These findings align with others suggesting a link between extraversion and mate preferences. Welling et al. (2008) found that extraverted women have a heightened preference for masculine male faces, a fitness cue. This preference could serve to mitigate inherently higher pathogen risks associated with their less restricted interpersonal style, which may be specific for a mating domain. If one can accurately identify extraversion while associating it with greater interest in promiscuous sexual strategies, a preference for introversion stemming from perceived infectability may function to reduce one's likelihood of contracting sexually transmitted infections. Although theoretically sensible, future research should determine the reliability of this proposed sex-specific effect.

Interestingly, and independent of pathogen concerns (both acute and dispositional), participants demonstrated a greaterthan-chance preference for extraversion, relative to introversion. This effect was driven primarily by participants' preference for extraversion in female target faces (relative to introversion). Despite viewing extraverted individuals as better able to satisfy affiliative needs, another set of communicated costs and benefits for men and women may emerge that make extraversion in women more desirable than it is for men. One possible reason for our results may be the perception that extraverted men are more likely to engage in extra-pair relations than are women, therefore potentially being seen as less trustworthy (Nettle 2005). Future research should identify individuals' perceptions of extraverted faces further to determine the influence of target sex. Nonetheless, extraversion appears desirable, particularly in female faces, and the fact that dispositional disease concerns down-regulate this preference suggests that individuals are acutely aware of both the opportunities and costs of others' extraversion and weigh each based on their own perceived infec tability.

Contrary to our hypotheses and past research, acute activation of disease threat did not influence face preferences (e.g., Young et al. 2011), nor did individual differences in perceived vulnerability to disease (germ aversion, perceived infectability) interact with acute disease activation to influence extraversion face preferences (e.g., Mortensen et al. 2010). Importantly, the non-significant finding for acute disease activation on face preferences does not appear to be a failure of manipulation, as disease-primed participants reported significantly greater disease concerns than did those in the control condition (as indexed by manipulation check questions). It is possible that in the context of extraversion preferences based on facial cues, dispositional disease concerns exert more influence than acute activation of disease concerns. Nonetheless, future research may benefit by utilizing different manipulations of disease salience to determine their potential to influence preferences for faces communicating introversion versus extraversion.

\section{Future Directions}

Although the effects sizes of this study's findings were relatively small, they were consistent with theory and hypotheses; these results should thus be interpreted with caution. The findings only provide limited support for an evolutionary theory of extraversion preference, as our disease manipulation did not influence extraversion preferences. Nonetheless, future research would benefit by determining the robustness of these findings. Extraversion communicated in human faces may provide valuable social information from which one can determine the extent to affiliate with a social target. Our results suggest that dispositional pathogen concerns facilitate preference shifts away from extraversion.

Other orthogonal motives could similarly influence face preferences. Assuming disease avoidance and affiliation motives work in hydraulic opposition to each other (Sacco, Young, and Hugenberg 2014), greater affiliative concerns should augment preferences for extraverted faces because 
such individuals would likely provide greater opportunities to satisfy such needs. If individuals motivated to avoid pathogens are more likely to emphasize the costs of affiliation with an extraverted individual, then individuals motivated by affiliation should prefer extraverted individuals. Future research could investigate this preference both dispositionally, as indexed by need to belong, or through social exclusion (i.e., situational activation of affiliative motives).

It is also important to note that other individual differences could also elicit reduced preferences for facially communicated extraversion. Given that participants may have been responding as if they were selecting mates, future studies should consider what kind of infections might have been the primary concern for high-PI individuals. Specifically, participants may have been considering the infectability of extraverted cross-sex targets in terms of sexually transmitted infections rather than disease in general. Future studies should investigate the basis of participants' disease-related aversion based on individual differences in disgust sensitivity (Tybur, Lieberman, and Griskevicius 2009). Disgust sensitivity addresses how averse someone is to something in the environment. Two domains of disgust in this model address disease in both the general pathogen context and one specific to disease in sexual domains (i.e., STIs). If participants were viewing cross-sex faces as possible mates, sexual disgust may predict this aversion to extraverted persons more clearly, relative to the more general pathogen disgust, as sexual disgust would likely operate as a function of one's aversion to contracting a sexually transmitted infection.
Another possibility to investigate temporal activation of pathogen-avoidant motives could be through individuals' most recent illness. Miller and Maner (2011) indicated that BIS-related aversion to potential disease vectors operates linearly with one's illness recency. Perhaps downregulating extraversion preferences may be highest for individuals who are immunologically weakest, as they may be most likely to contract illness transmitted by gregariousness at that moment. Future studies should consider individuals' most recent illnesses in how they perceive others in their attempt to prevent further infection.

\section{Conclusion}

This study contributes to the corpus of person perception literature by identifying how faces communicating varying degrees of extraversion produce differential preferences. What makes this contribution unique is its investigation of preferences as a function of disease concern. The behavioral immune system has a pervasive influence on interpersonal behavior that its responses to disease threat can ultimately determine humans' decisions to approach or avoid someone by virtue of perceiving that person's proclivity to engage with others socially.

\section{Appendix}

Fig. 3 Example facial stimuli
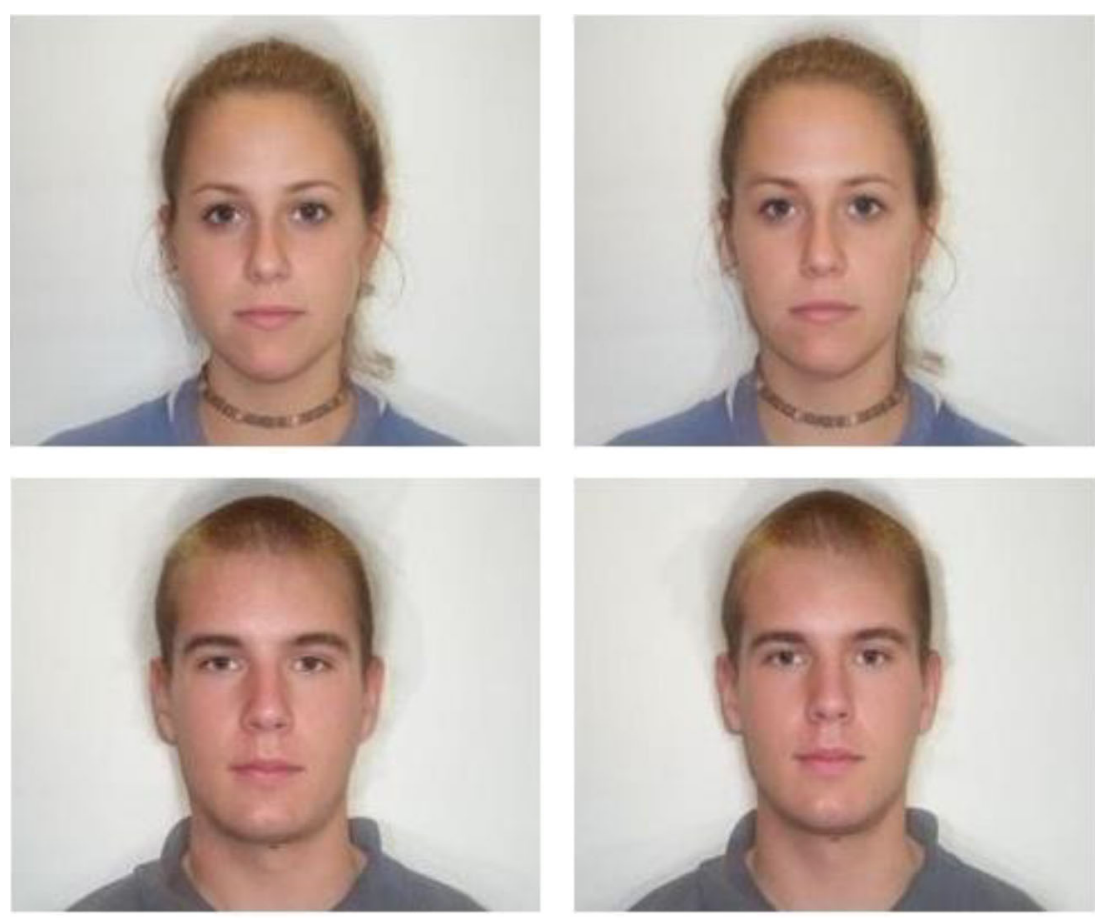

Faces communicating extraversion (left) and introversion (right) 


\section{References}

Ackerman, J. M., Becker, D. V., Mortensen, C. R., Sasaki, T., Neuberg, S. L., \& Kenrick, D. T. (2009). A pox on the mind: disjunction of attention and memory in the processing of physical disfigurement. Journal of Experimental Social Psychology, 45, 478-485.

Borkenau, P., Brecke, S., Möttig, C., \& Paelecke, M. (2009). Extraversion is accurately perceived after a 50-ms exposure to a face. Journal of Research in Personality, 43, 703-706.

Buhrmester, M., Kwang, T., \& Gosling, S. D. (2011). Amazon's Mechanical Turk: a new source of inexpensive, yet high-quality, data? Perspectives on Psychological Science, 6, 3-5.

Duncan, L. A., \& Schaller, M. (2009). Prejudicial attitudes toward older adults may be exaggerated when people feel vulnerable to infectious disease: evidence and implications. Analyses of Social Issues and Public Policy, 9, 97-115.

Duncan, L. A., Schaller, M., \& Park, J. H. (2009). Perceived vulnerability to disease: development and validation of a 15-item self-report instrument. Personality and Individual Differences, 47, 541-546.

Faul, F., Erdfelder, E., Lang, A. G., \& Buchner, A. (2007). G* Power 3: a flexible statistical power analysis program for the social, behavioral, and biomedical sciences. Behavior Research Methods, 39, 175-191.

Gangestad, S. W., \& Buss, D. M. (1993). Pathogen prevalence and human mate preferences. Ethology and Sociobiology, 14, 89-96.

Gangestad, S. W., Thornhill, R., \& Yeo, R. A. (1994). Facial attractiveness, developmental stability, and fluctuating asymmetry. Ethology and Sociobiology, 15, 73-85.

Halperin, D. T., \& Epstein, H. (2004). Concurrent sexual partnerships help to explain Africa's high HIV prevalence: implications for prevention. The Lancet, 364, 4-6.

Hamrick, N., Cohen, S., \& Rodriguez, M. S. (2002). Being popular can be healthy or unhealthy: stress, social network diversity, and incidence of upper respiratory infection. Health Psychology, 21, 294 298.

Hayes, A. F. (2013). Introduction to mediation, moderation, and conditional process analysis: a regression-based approach. New York: Guilford Press.

Holtzman, N. S. (2011). Facing a psychopath: detecting the dark triad from emotionally-neutral faces, using prototypes from the personality Faceaurus. Journal of Research in Personality, 45, 648-654.

Little, A. C., DeBruine, L. M., \& Jones, B. C. (2011). Exposure to visual cues of pathogen contagion changes preferences for masculinity and symmetry in opposite-sex faces. Proceedings of the Royal Society of London B: Biological Sciences, 278, 2032-2039.

Little, A. C., \& Perrett, D. I. (2007). Using composite images to assess accuracy in personality attribution to faces. British Journal of Psychology, 98, 111-126.

Ma, D. S., Correll, J., \& Wittenbrink, B. (2015). The Chicago face database: a free stimulus set of faces and norming data. Behavior Research Methods, 47, 1122-1135.

Miller, S. L., \& Maner, J. K. (2011). Sick body, vigilant mind the biological immune system activates the behavioral immune system. Psychological Science, 22, 1467-1471.

Miller, S. L., \& Maner, J. K. (2012). Overperceiving disease cues: the basic cognition of the behavioral immune system. Journal of Personality and Social Psychology, 102, 1198-1213.
Minear, M., \& Park, D. C. (2004). A lifespan database of adult facial stimuli. Behavior Research Methods, Instruments, \& Computers, 36, 630-633.

Mortensen, C. R., Becker, D. V., Ackerman, J. M., Neuberg, S. L., \& Kenrick, D. T. (2010). Infection breeds reticence: the effects of disease salience on self-perceptions of personality and behavioral avoidance tendencies. Psychological Science, 21, 440-447.

Murray, D. R., Jones, D. N., \& Schaller, M. (2013). Perceived threat of infectious disease and its implications for sexual attitudes. Personality and Individual Differences, 54, 103-108.

Nettle, D. (2005). An evolutionary approach to the extraversion continuum. Evolution and Human Behavior, 26, 363-373.

Neuberg, S. L., Kenrick, D. T., \& Schaller, M. (2011). Human threat management systems: self-protection and disease avoidance. Neuroscience \& Biobehavioral Reviews, 35, 1042-1051.

Petrican, R., Todorov, A., \& Grady, C. (2014). Personality at face value: facial appearance predicts self and other personality judgments among strangers and spouses. Journal of Nonverbal Behavior, 38 , 259-277.

Pollett, T. V., Roberts, S. G., \& Dunbar, R. I. (2011). Extraverts have larger social network layers. Journal of Individual Differences, 32, 161-169.

Sacco, D. F., Young, S. G., \& Hugenberg, K. (2014). Balancing competing motives: adaptive trade-offs are necessary to satisfy disease avoidance and interpersonal affiliation goals. Personality and Social Psychology Bulletin, 40, 1611-1623.

Schaller, M., Miller, G. E., Gervais, W. M., Yager, S., \& Chen, E. (2010). Mere visual perception of other people's disease symptoms facilitates a more aggressive immune response. Psychological Science, $21,459-462$.

Schaller, M., \& Murray, D. R. (2008). Pathogens, personality, and culture: disease prevalence predicts worldwide variability in sociosexuality, extraversion, and openness to experience. Journal of Personality and Social Psychology, 95, 212-221.

Schaller, M., \& Duncan, L. A. (2007). The behavioral immune system: Its evolution and social psychological implications. In J. P. Forgas, M. G. Haselton, \& W. von Hippel (Eds.), Evolution and the Social Mind: Evolutionary Psychology and Social Cognition (pp. 293307). New York: Psychology Press.

Schmitt, D. P., \& Shackelford, T. K. (2008). Big five traits related to shortterm mating: from personality to promiscuity across 46 nations. Evolutionary Psychology, 6, 246-282.

Thornhill, R., Fincher, C. L., Murray, D. R., \& Schaller, M. (2010). Zoonotic and non-zoonotic diseases in relation to human personality and societal values: support for the parasite-stress model. Evolutionary Psychology, 8, 151-169.

Tybur, J. M., Lieberman, D., \& Griskevicius, V. (2009). Microbes, mating, and morality: individual differences in three functional domains of disgust. Journal of Personality and Social Psychology, 97, 103122.

Welling, L. L. M., DeBruine, L. M., Little, A. C., \& Jones, B. C. (2008). Extraversion predicts individual differences in women's face preferences. Personality and Individual Differences, 47, 906-908.

Young, S. G., Sacco, D. F., \& Hugenberg, K. (2011). Vulnerability to disease is associated with a domain-specific preference for symmetrical faces relative to symmetrical non-face stimuli. European Journal of Social Psychology, 41, 558-563. 